\title{
1 CBRPP: a new RNA-centric method to study RNA-protein interactions
}

3 Yunfei $\mathrm{Li}^{1}$, Shengde Liu ${ }^{1}$, Lili Cao ${ }^{1}$, Yujie Luo ${ }^{1}$, Hongqiang Du ${ }^{1}$, Siji Li ${ }^{1}$, Fuping You ${ }^{1, \#}$

$4{ }^{1}$ Institute of Systems Biomedicine, Department of Immunology, School of Basic Medical 5 Sciences, Beijing Key Laboratory of Tumor Systems Biology, Peking University Health Science 6 Center, Beijing, China.

$7{ }^{\#}$ Corresponding authors: Fuping You Ph.D, Institute of Systems Biomedicine, Department of 8 Pathology, School of Basic Medical Sciences, Beijing Key Laboratory of Tumor Systems Biology,

$9 \quad$ Peking University Health Science Center, Beijing, China. fupingyou@ hsc.pku.edu.cn. 


\section{Abstract}

11 RNA-protein interactions play essential roles in tuning gene expression at RNA level and 12 modulating the function of proteins. Abnormal RNA-protein interactions lead to cell dysfunction 13 and human diseases. Therefore, mapping networks of RNA-protein interactions is crucial for 14 understanding cellular mechanism and pathogenesis of diseases. Different practical protein-centric 15 methods for studying RNA-protein interactions has been reported, but few RNA-centric methods 16 exist. Here, we developed CRISPR-based RNA proximity proteomics (CBRPP), a new 17 RNA-centric method to identify proteins associated with the target RNA in native cellular context 18 without cross-linking or RNA manipulation in vitro. CBRPP is based on a fusion of dCas13 and 19 proximity-based labeling (PBL) enzyme. dCas13 can deliver PBL enzyme to the target RNA with 20 high specificity, while PBL enzyme labels the surrounding proteins of the target RNA, which are 21 then identified by mass spectrometry.

22 Keywords: RNA-protein interactions, dPspCas13b, dRfxCas13d, APEX2, TurboID, BASU, 23 BioID2, CBRPP 


\section{Introduction}

RNA is bound to protein from birth to death. RNA-binding proteins (RBPs) play a pivotal role in a wide range of biological processes, including RNA transcription, processing, modification, transport, translation and stabilization ${ }^{[1-4]}$. RNAs, in turn, influence proteins expression, localization and interactions with other proteins ${ }^{[5-7]}$. Aberrant RNA-protein interactions are related to cellular dysfunction and human diseases ${ }^{[3,8,9]}$. Therefore, mapping networks of RNA-protein interactions is of great importance for understanding many cellular biological processes.

Based on the type of molecule they start with, methods for studying RNA-protein interactions are classified into protein-centric methods and RNA-centric methods ${ }^{[10]}$. Protein-centric methods start with a protein of interest and study RNAs that interact with that protein. Since proteins are easily purified with antibodies, many protein-centric methods, such as cross-linking immunoprecipitation (CLIP)-seq ${ }^{[11]}$ and RNA immunoprecipitation (RIP)-seq ${ }^{[12]}$, are available and practical. Conversely, RNA-centric methods start with an RNA of interest and focus on proteins that bind it. Most approaches use biotinylated RNA ${ }^{[13]}$, aptamer-tagged RNA ${ }^{[14]}$, peptide nucleic acid $^{[15]}$ and antisense probe ${ }^{[16-20]}$ for purification of RNA-protein complexes to identify proteins that associate with the target RNA, however these methods often require RNA manipulation in vitro and miss transient or weak interactions. Meanwhile, compared with protein-centric methods, there are few robust RNA-centric methods.

In this paper, by combining the power of CRISPR-Cas $13^{[21]}$ and proximity-based labeling (PBL) technique $^{[22]}$, we developed CBRPP (CRISPR-based RNA proximity proteomics), a new RNA-centric method to identify proteins associated with the target RNA in native cellular context without cross-linking or RNA manipulation in vitro. 


\section{$47 \quad$ Results}

48

49

50

51

52

53

54

55

56

57

58

59

60

61

62

63

64

65

66

67

68

69

70

71

72

73

74

75

76

77

78

79

80

81

82

83

84

\section{Strategies to develop CBRPP}

In recent years, PBL has emerged as a powerful complementary approach to classic affinity purification of multiprotein complexes in mapping of protein-protein interactions ${ }^{[23]}$. By fusing proteins of interest to enzymes that generate reactive molecules, most commonly biotin, adjacent proteins are covalently labeled so that they can be isolated and identified ${ }^{[22]}$. To date, multiple versions of the PBL enzyme have been developed, such as BioID2 ${ }^{[24]}$, TurboID ${ }^{[25]}$, Apex $2^{[26]}$ and $\mathrm{BASU}^{[27]}$. The key advantage of PBL is that it can capture weak and transient interactions in live cells. Recently, two studies have applied PBL to study RNA-protein interactions using the MS2-MCP strategy ${ }^{[28]}$ or a similar strategy ${ }^{[27]}$, but both require insertion of MS2 or BoxB stem-loop into the target RNA in advance, which may influence structure or function of the target RNA.

The discovery of RNA-targeting CRISPR systems offers scientists a powerful toolbox to manipulate RNA in live cells ${ }^{[21]}$. Active Cas13, under the guidance of the specific CRISPR RNA (crRNA), can recognize and cleave the target RNA. Catalytically dead Cas13 (dCas13) retains programmable RNA-binding capability, which can be utilized for RNA imaging and editing ${ }^{[29,30]}$. Currently, there are several orthologs and subtypes of Cas13 that are catalytically active inside mammalian cells, including LwaCas13a ${ }^{[29]}, P_{s p C a s} 13 b^{[30]}$ and RfxCas $13 \mathrm{~d}^{[31]}$. Inspired by GLoPro $^{[32]}$ and C-BERST ${ }^{[33]}$, we proposed that by fusing dCas13 and PBL enzyme together, dCas13, under the guidance of a specific crRNA, can act as an RNA tracker to bring PBL enzyme to the target RNA, then PBL enzyme can biotinylate the surrounding proteins of the target RNA with biotin. Finally, these biotinylated proteins can be easily enriched by streptavidin beads and identified by liquid chromatography mass spectrometry (LC-MS) (Figure 1). We referred to this combination of CRISPR-Cas13 and PBL as CRISPR-based RNA proximity proteomics (CBRPP).

\section{dRfxCas13d is not suitable for CBRPP to study RNA-protein interactions}

To prove the concept, we firstly selected $\mathrm{dRfxCas} 13 \mathrm{~d}$ and APEX2 for testing, because RfxCas13d is the smallest and most active one among Cas13 proteins ${ }^{[31]}$ and APEX2 have the fastest rate of labeling ${ }^{[26]}$, which can be used for isolated analysis of RNA-protein interactions that occur over short time periods. We fused APEX2 to N-terminus or C-terminus of RfxCas13d to test whether the fusion of APEX2 affected the function of RfxCas13d by detecting knockdown efficiency of RfxCas13d (Figure 2A). Results showed that fusion of APEX2 to C-terminus of RfxCas13d only slightly affect the knockdown efficiency of RfxCas13d, and has no effect on the expression of RfxCas13d (Figure 2B). Therefore, we constructed dRfxCas13d-APEX2-NES plasmid (Figure 2A) and applied it to well-studied ACTB mRNA to test whether it would identify known RBPs of ACTB mRNA. We designed seven Rfx-crRNAs targeting different regions of ACTB mRNA and validated their targeting by knockdown with an active RfxCas13d. RT-qPCR results showed that all seven Rfx-crRNAs significantly reduced ACTB mRNA levels in HEK293T cells (Figure 2C). Then we transfected HEK293T with dRfxCas13d-APEX2-NES and two optimal 
ACTB Rfx-crRNAs (crRNA4 and crRNA7) to test whether dRfxCas13d-APEX2-NES can be directed to ACTB mRNA under the guidance of ACTB RfX-crRNAs. In addition, cells were treated with sodium arsenite to induce the formation of stress granules where ACTB mRNA accumulated. Results showed that dRfxCas13d-APEX2-NES could colocalize with the stress granule marker G3BP1 regardless of co-transfection with ACTB Rfx-crRNAs or non-targeting Rfx-crRNAs (Figure 2D). This indicated that dRfxCas13d-APEX2-NES may nonspecifically accumulate with ACTB mRNA. We also constructed dRfxCas13d-APEX2-NLS plasmid and designed the Rfx-crRNAs targeting NEAT1 to study paraspeckles. We found that the localization of dRfxCas13d-APEX2-NLS had no difference between the non-targeting crRNA group and the NEAT1 targeting crRNA group (data not show), which is consistent with results of Chen lab ${ }^{[34]}$. These data suggested that dRfxCas13d is not suitable for CBRPP to study RNA-protein interactions.

\section{Transient transfection of dPspCas13b-APEX2 to study RBPs of ACTB mRNA is not effective}

Recent study showed that $\mathrm{dPspCas} 13 \mathrm{~b}$ is the most efficient dCas13 protein to label $\mathrm{RNA}^{[34]}$, so we replaced dRfxCas13d with dPspCas13b and added a flexible linker 3x(GGGGS) between dPspCas13b and APEX2 to avoid mutual influence (Figure 3A). Since PspCas13b and RfxCas13d cannot share the crRNAs, we redesigned four ACTB Psp-crRNAs and validated their targeting. Results showed that all four ACTB Psp-crRNAs significantly reduced ACTB mRNA levels in HEK293T cells, and that the knockdown efficiency and expression level were comparable between PspCas13b and PspCas13b-APEX2 (Figure 3B and 3C). Furthermore, co-transfection of dPspCas13b-APEX2 and ACTB Psp-crRNAs in HEK293T did not affect the mRNA and protein level of ACTB (Figure 3D), suggesting this system does not affect the stability and translation of ACTB mRNA. Then, we transiently transfected dPspCas13b-APEX2 and ACTB Psp-crRNAs into HEK293T cells to performed a 1-minute proximity labeling reaction, followed by streptavidin bead enrichment of biotinylated proteins and LC-MS (Figure 3E). The streptavidin-HRP blot showed that dPspCas13b-APEX2 has biotinylation activity (Figure 3F). Mass spectrometry profiling results showed that the known RBPs of ACTB mRNA (marked in red) were not enriched in the ACTB Psp-crRNA group relative to the non-targeting Psp-crRNA group (Figure 3G). These data suggested that transient transfection of dPspCas13b-APEX2 to study RBPs of ACTB mRNA is not effective.

We speculated that such results may be due to the high expression of dPspCas13b-APEX2 or the properties of APEX2 itself. If the protein expression level of dPspCas13b-APEX2 is too high, or the copy number of dPspCas13b-APEX2 proteins exceeds that of target RNAs, some redundant dPspCas13b-APEX2 proteins cannot be directed to the target RNAs with the guidance of specific crRNAs, so background proteins would be labeled, resulting in low signal-to-noise ratio. It's known that APEX2-based labeling is often specific to low-abundance amino acids such as tyrosine $^{[35,36]}$, so it is possible that labeling will not occur if surface-exposed tyrosine is not available.

\section{Inducible expression of dPspCas13b-BioID2 successfully identifies RBPs of}




\section{ACTB mRNA}

For further optimization, we next used other three PBL enzymes (BioID2, TurboID and BASU) to test which enzyme is optimal (Figure 4A). Simultaneously, we took advantage of the Tet-On 3G inducible expression system to keep the expression of fusion proteins at a low level in HEK293T cells (Supplementary Figure 1). RT-qPCR results showed that the knockdown efficiency of PspCas13b was not affected by fusion BioID2/TurboID/BASU/APEX2 to C terminus of PspCas13b (Figure 4B). Therefore, we constructed four stable HEK293T cell lines for inducible expression of dPspCas13b- BioID2/TurboID/BASU/Apex2. Western blotting results showed that all four stable cell lines can be induced by doxycycline in a dose-dependent manner, and that BioID2, TurboID and APEX2 have biotinylation activity but not BASU (Figure 4C). Subsequently, we used dPspCas13b-BioID2/TurboID/Apex2 inducibly expressing cell lines to identify the proteins interacting with ACTB mRNA (Figure 4D and 4E). We analyzed the protein mass spectrometry data obtained from these three cell lines, and found that in dPspCas13b-BioID2 inducibly expressing cell line, the known RBPs of ACTB mRNA (marked in red) such as IGF2BP1, HNRNPA1, HNRNRC, HNRNPA2B1 and HNRNPM, were significantly enriched in ACTB Psp-crRNA group relative to the non-targeting Psp-crRNA group (Figure 4F and Supplementary Figure 2). IGF2BP1, also known as ZBP1 (zipcode-binding protein 1), interacts with the zipcode of ACTB mRNA via KH (HNRNPK homology) domains to regulate the localization and translation of ACTB $\mathrm{mRNA}^{[37]}$. HNRNPA1, HNRNPC, HNRNPA2B1 and HNRNPM are common RBPs that are involved not only in processing heterogeneous nuclear RNAs (hnRNAs) into mRNAs, but also mRNAs stability and translational regulation ${ }^{[38]}$. KHSRP have been suggested to be associated with ACTB mRNA localization ${ }^{[39]}$. These data indicated that inducible expression of dPspCas13b-BioID2 successfully identify RBPs of ACTB mRNA.

Unlike TurboID or APEX2, BioID2 used in CBRPP generates a history of RNA-protein interactions over time, which can capture some transient RNA-protein interactions, such as those occur during various stages of the cell cycle. Besides, the results obtained using BioID2 in CBRPP represent the accumulation of biotinylated proteins over the labeling time. The proteins that interact with the target RNA are labeled and accumulated during this time, and those background proteins that occasionally appear near the target RNA without mutual interaction may be labeled but not accumulated, which result in high signal-to-noise ratio. Therefore, inducible expression of dPspCas13b-BioID2 is recommended to study RBPs of the target RNA.

\section{Discussion}

Here we proposed a new RNA-centric method named CBRPP by combining dCas 13 with proximity-based labeling. With some optimizations, we finally determined that inducible expression of dPspCas13b-BioID2 is most suitable for studying RNA-protein interactions. In the presence of a specific crRNA, the dPspCas13b-BioID2 fusion protein is directed to the target RNA, then BioID2 in the chimera biotinylates nearby proteins of the target RNA. With the strong interaction between biotin and streptavidin, biotinylated proteins can be easily enriched and identified. 

does not require pre-labeling of the target $\mathrm{RNA}^{[13]}$, MS2 insertion in advance ${ }^{[28]}$, or designing 166 antisense probes ${ }^{[16-20]}$ to purify RNA-protein complexes. In dPspCas13b-BioID2 positive cells 167 only crRNAs are required. Second, using CBRPP, RBPs labeling is done in a living cell state 168 without manipulating RNA-protein complexes in vitro, so it almost preserves the natural structure 169 of the target RNA, while avoiding the possible disruption of RNA-protein interactions and RNA 170 degradation. Third, CBRPP can capture weak and transient RNA-protein interactions, taking 171 advantage of proximity-based labeling ${ }^{[22]}$.

As with any technology, CBRPP has its limitations. Since proximity-based labeling is in a distance-dependent manner, proteins identified by CBRPP may be not RBPs of the target RNA but merely proximate proteins. Therefore, it is necessary to confirm the interactions between the target RNA and candidate proteins identified by CBRPP with RIP or CLIP. Due to the large size of dPspCas13b-BioID2, its binding to the target RNA may affect the binding of the original interacted protein at this site. In addition, the long labeling time required for BioID2 methods prevents CBRPP from isolated analysis of RNA-protein interactions that occur over short period of time.

According to our experience, there are three crucial factors for the success of CBRPP. First, it is necessary to find potent crRNAs for analysis, and testing multiple crRNAs at the same time is recommended. Second, the expression level of dPspCas13b-BioID2 should be controlled at a low level in case the copy number of fusion proteins exceeds that of the target RNAs, resulting in low

184 signal-to-noise ratio. Third, setting up an appropriate control group is very helpful for excluding 185 background proteins identified by the experimental group.

In summary, in this study we developed an effective RNA centric method to identify proteins associated with the target RNA in native cellular context without cross-linking or RNA manipulation in vitro. Although we have only studied ACTB mRNA using CBRPP, in principle CBRPP can also be used to study lncRNA or other RNA types. For large lncRNA, taking Xist as an example, by designing different crRNAs target different regions of Xist, CBRPP can not only study the RBPs of Xist, but also the RBPs at a certain position of $\mathrm{Xist}^{[16,40]}$. Furthermore, CBRPP

192 is suitable for studying the mechanism of diseases caused by abnormal RNA, such as myotonic 193 dystrophy type $1^{[7]}$. 


\section{Materials and Methods}

196

197

198

199

200

201

202

203

204

205

206

207

208

209

210

211

212

213

214

215

216

217

218

219

220

221

222

223

224

225

226

227

\section{Cell culture}

HEK293T (Human Embryonic Kidney 293T) cells was obtained from ATCC. Cells were cultured in DMEM medium supplemented with 10\% FBS (Gibco) and 100U/ml Penicillin-Streptomycin in a humidified incubator at $37{ }^{\circ} \mathrm{C}$ with $5 \% \mathrm{CO}_{2}$.

\section{Reagents and Antibodies}

PEI (764582, Sigma-Aldrich) was used for transfection. Antibodies used in this study include the following: anti-HA (rabbit, H6908, Sigma-Aldrich); anti-alpha-tubulin (rabbit, 11224-1-AP, Proteintech); anti-G3BP1 (mouse, sc-365338, Santa Cruz); HRP-conjugated Streptavidin (SA00001-0, Proteintech). The antibodies were diluted 1,000 times for immunoblots, 200 times in confocal microscopy.

\section{Plasmid constructs}

Expression constructs generated for this study were prepared by standard molecular biology techniques and coding sequences entirely verified. All the mutants were constructed by standard molecular biology technique. Each mutant was confirmed by sequencing. All plasmid constructs and their sequence were listed in Supplementary Table 1. All crRNAs used in this paper were listed in Supplementary Table 2.

\section{Western blotting}

Cells were washed with PBS and lysed by incubation on ice for 10 min with RIPA lysis buffer (50 mM Tris, $150 \mathrm{mM} \mathrm{NaCl}, 0.1 \% \mathrm{SDS}, 0.5 \%$ sodium deoxycholate, $1 \%$ Triton X-100, protease cocktail [C0001, Targetmol], and $1 \mathrm{mM}$ PMSF). The proteins were resolved by SDS/PAGE and transferred to 0.22 um nitrocellulose membrane (PALL), which then was incubated overnight with primary antibodies. The membrane was further incubated with the corresponding HRP-conjugated secondary antibodies and detected by enhanced chemiluminescence.

\section{Immunofluorescence microscopy}

HEK293T cells were plated and grew on coverslips with indicated treatments, washed with pre-warmed PBS, and fixed with $4 \%$ paraformaldehyde for $10 \mathrm{~min}$. The cells were permeated with $0.5 \%$ Triton-100 for $3 \mathrm{~min}$, blocked with 3\% BSA for $30 \mathrm{~min}$, washed, and incubated with primary antibodies for $1 \mathrm{~h}$ at $37{ }^{\circ} \mathrm{C}$. After washing, cells were stained with Alexa Fluor 488-conjugated secondary antibodies (A11029, Invitrogen) or Alexa Fluor 555-conjugated secondary antibodies (A-21428, Invitrogen) for $1 \mathrm{~h}$ at $37^{\circ} \mathrm{C}$, and then with DAPI (4',6-Diamidino-2-phenylindole, Roche) for $15 \mathrm{~min}$. The coverslips were washed extensively and mounted onto slides. Imaging of the cells were carried out using N-STORM5.0 microscope. 


\section{RNA Extraction and Quantitative reverse transcription PCR (RT-qPCR)}

Total RNA from cells were isolated using the RNA simple Total RNA kit (TIANGEN). 1ug RNA was reverse transcribed using a FastKing RT Kit (TIANGEN). Levels of the indicated genes were analyzed by quantitative real-time PCR amplified using SYBR Green (Q311, Vazyme). All primers were listed in Supplementary Table 3.

\section{Generation of Stable Expression Mammalian Cell Lines}

For preparation of lentiviruses, HEK293T cells in 6-well plates were transfected with the lentiviral vector of interest $(1,800 \mathrm{ng})$, the lentiviral packaging plasmids psPAX2 (600 ng) and pMD2.G (600 ng) and $12 \mathrm{ul}$ of PEI $(1 \mathrm{mg} / \mathrm{ml})$. About $48 \mathrm{~h}$ after transfection the cell medium containing lentiviruses was centrifugalized at $12,000 \mathrm{~g}$ for 3 minutes and the supernatant was harvested. HEK293 cells were then infected at $\sim 50 \%$ confluency by lentiviruses for $48 \mathrm{~h}$, followed by selection with $1 \mu \mathrm{g} / \mathrm{ml}$ puromycin in growth medium for 7 days. The stable transgene monoclonal cells were harvested by limiting dilution in cell pools.

\section{Generation of Tetracycline (Tet) Inducible Expression HEK293T cell lines}

The two consecutive manipulation steps are necessary to generate human Tet-on cell lines with inducible expression of plasmids of interest. The first step is generation of cells stably expressing reverse tetracycline-controlled transactivator (rtTA). HEK293T cells were infected at $\sim 50 \%$ confluency by lentiviruses containing pLVX-TetO3G(rtTA)-hygr vector for $48 \mathrm{~h}$, followed by selection with $50 \mathrm{ug} / \mathrm{ml}$ hygromycin in growth medium for 7 days, and hygromycin resistant clones were selected. Several clones were picked and tested for rtTA expression by immunoblotting. After testing for all molecular and cell biological parameters of interest, the 'best' rtTA-positive clone was expanded and stored. The next step is generation of Tet-on cell lines with inducible expression of target plasmids. The 'best' rtTA-positive clone was infected by lentiviruses containing target plasmids (Inducible-dPspCas13b-BioID2/BASU/TurboID/APEX2) for $48 \mathrm{~h}$, followed by selection with $1 \mu \mathrm{g} / \mathrm{ml}$ puromycin in growth medium for 7 days. The puromycin resistant clones were harvested by limiting dilution in cell pools. Several individual cell clones were picked, expanded and screened by immunoblotting for Doxycycline-inducible expression of the gene of interest. Finally, clones of interest were expanded, re-tested and stored.

\section{Biotin Labeling in Live Cells}

For dPspCas13b-APEX2 transient transfection experiments, HEK293T cells were plated in 10 $\mathrm{cm}$ dish at $70 \%$ confluency 18 hours prior to transfection. Cells were transfected with the dPspCas13b-Apex 2 plasmid and the crRNA plasmids. After 6 hours of transfection, the culture medium was changed. After 24 hours of transfection, biotin-phenol was added to cell culture medium to a final concentration of $500 \mathrm{uM}$ for 30 minutes, $\mathrm{H}_{2} \mathrm{O}_{2}$ was then added into cell culture media at a final concentration of $1 \mathrm{mM}$ to induce biotinylation. After gently shaking the cell culture dish for one minute, the medium was removed and cells were washed three times with PBS supplemented with $100 \mathrm{mM}$ sodium azide, $100 \mathrm{mM}$ sodium ascorbate and 50mM TROLOX. Cells 
were scraped and transferred to $1.5 \mathrm{ml}$ tubes with ice cold PBS, spun at $3600 \mathrm{rpm}$ for 5 minutes, flash frozen in liquid nitrogen and stored at $-80^{\circ} \mathrm{C}$.

For inducibly expressing dPspCas13b-BioID2/TurboID/BASU/Apex2 experiments, four stable HEK293T cell lines for inducible expression of dPspCas13b- BioID2/TurboID/BASU/Apex2 were plated in $10 \mathrm{~cm}$ dish at $70 \%$ confluency 18 hours prior to transfection. Cells were transfected with 20ug crRNA plasmid per dish. After 6 hours of transfection, the culture medium was replaced with new media containing $0.1 \mathrm{ug} / \mathrm{ml}$ doxycycline. For BioID2, biotin was added to the culture medium at a final concentration of $50 \mathrm{uM}$ after 15 hours of transfection; for TurboID, biotin was added at a final concentration of $500 \mathrm{uM}$ for 10 minutes before harvesting cells; for BASU, biotin was added at a final concentration of 200uM for 2 hours before harvesting cells; for APEX2, biotin-phenol was added at a final concentration of $500 \mathrm{uM}$ for 30 minutes and $\mathrm{H}_{2} \mathrm{O}_{2}$ was added at a final concentration of $1 \mathrm{mM}$ for one minute before harvesting cells. All kinds of cells were harvested at 33 hours after transfection. For APEX2, the medium was removed and cells were washed three times with ice cold PBS supplemented with $100 \mathrm{mM}$ sodium azide, $100 \mathrm{mM}$ sodium ascorbate and 50mM TROLOX; for TurboID/BASU/BioID2, the medium was removed and cells were washed three times with ice cold PBS. Cells were scraped and transferred to $1.5 \mathrm{ml}$ tubes with ice cold PBS, spun at $3600 \mathrm{rpm}$ for 5 minutes, flash frozen in liquid nitrogen and stored at $-80^{\circ} \mathrm{C}$.

\section{Streptavidin Magnetic Bead Enrichment of Biotinylated Proteins}

Cell pellets as described above were lysed in RIPA lysis buffer (50 mM Tris, $150 \mathrm{mM} \mathrm{NaCl}, 0.1 \%$ SDS, $0.5 \%$ sodium deoxycholate, $1 \%$ Triton X-100, protease cocktail [TargetMol], and $1 \mathrm{mM}$ PMSF) at $4{ }^{\circ} \mathrm{C}$ for 10 minutes. The lysates were cleared by centrifugation at $12,000 \mathrm{~g}$ for $10 \mathrm{~min}$ at $4{ }^{\circ} \mathrm{C}$. 50ul of each lysate supernatant was reserved for detection of biotinylation activity by western blotting. Streptavidin magnetic beads were washed twice with RIPA lysis buffer and then mixed with lysates supernatant together with rotation overnight at $4{ }^{\circ} \mathrm{C}$. On day 2 , the beads were subsequently washed twice with $1 \mathrm{~mL}$ of RIPA lysis buffer, once with $1 \mathrm{~mL}$ of $1 \mathrm{M} \mathrm{KCl}$, once with $1 \mathrm{~mL}$ of $0.1 \mathrm{M} \mathrm{Na}_{2} \mathrm{CO}_{3}$, once with $1 \mathrm{~mL}$ of $2 \mathrm{M}$ urea in $10 \mathrm{mM}$ Tris- $\mathrm{HCl}$ (pH 8.0), and twice with $1 \mathrm{~mL}$ RIPA lysis buffer. Finally, biotinylated proteins were eluted by boiling the beads in $150 \mu \mathrm{L}$ of elution buffer (55 mM pH 8.0 Tris-HCl, 0.1\% SDS, 6.66mM DTT, $0.66 \mathrm{mM}$ biotin) for 10 minutes and sent for mass spectrometry.

\section{Statistical Analysis}

The descriptive statistical analysis was performed with Prism version 7 (GraphPad Software). All data are presented as mean $\pm \mathrm{SD}$. A two-tailed Student's $t$ test assuming equal variants was used to compare two groups. In all figures, the statistical significance between the indicated samples and control is designated as $* \mathrm{P}<0.05, * * \mathrm{P}<0.01, * * * \mathrm{P}<0.001$, or NS $(\mathrm{P}>0.05)$. 


\section{Acknowledgments}

302 This work was supported by the National Natural Science Foundation of China (31570891) and

303 the National Key Research and Development Program of China (Grant \#2016YFA0500302).

\section{Author Contributions}

305 Y.L. and F.Y. conceived this project. Y.L. analyzed the data and wrote the paper. Y.L., SD.L., L.C.,

306 H.D. and F.Y. revised the paper. Y.L., SD.L., L.C. and YJ.L. performed most experiments. SJ.L.

307 contributed to imaging.

\section{Declaration of Interests}

309 The authors declare no competing interests. 


\section{Reference}

312 1. Lee SR, Lykke-Andersen J. Emerging roles for ribonucleoprotein modification and remodeling

313 in controlling RNA fate. Trends Cell Biol 2013; 23(10):504-510.

314 2. Muller-McNicoll M, Neugebauer KM. How cells get the message: dynamic assembly and 315 function of mRNA-protein complexes. Nat Rev Genet 2013; 14(4):275-287.

316 3. Gerstberger S, Hafner M, Tuschl T. A census of human RNA-binding proteins. Nat Rev Genet $3172014 ; 15(12): 829-845$.

318 4. Di Liegro CM, Schiera G, Di Liegro I. Regulation of mRNA transport, localization and

319 translation in the nervous system of mammals (Review). Int J Mol Med 2014; 33(4):747-762.

320 5. Bugaut A, Balasubramanian S. 5'-UTR RNA G-quadruplexes: translation regulation and 321 targeting. Nucleic Acids Res 2012; 40(11):4727-4741.

322 6. Ma W, Mayr C. A Membraneless Organelle Associated with the Endoplasmic Reticulum 323 Enables 3'UTR-Mediated Protein-Protein Interactions. Cell 2018; 175(6):1492-1506 e1419.

324 7. Jain A, Vale RD. RNA phase transitions in repeat expansion disorders. Nature 2017; 325 546(7657):243-247.

326 8. Baltz AG, Munschauer M, Schwanhausser B, Vasile A, Murakawa Y, Schueler M, et al. The 327 mRNA-bound proteome and its global occupancy profile on protein-coding transcripts. Mol Cell 328 2012; 46(5):674-690.

329 9. Castello A, Fischer B, Eichelbaum K, Horos R, Beckmann BM, Strein C, et al. Insights into RNA 330 biology from an atlas of mammalian mRNA-binding proteins. Cell 2012; 149(6):1393-1406.

331 10. Ramanathan M, Porter DF, Khavari PA. Methods to study RNA-protein interactions. Nat 332 Methods 2019; 16(3):225-234.

333 11. Licatalosi DD, Mele A, Fak JJ, Ule J, Kayikci M, Chi SW, et al. HITS-CLIP yields genome-wide 334 insights into brain alternative RNA processing. Nature 2008; 456(7221):464-469.

335 12. Nicholson CO, Friedersdorf M, Keene JD. Quantifying RNA binding sites transcriptome-wide 336 using DO-RIP-seq. RNA 2017; 23(1):32-46.

337 13. Zheng X, Cho S, Moon H, Loh TJ, Jang HN, Shen H. Detecting RNA-Protein Interaction Using 338 End-Labeled Biotinylated RNA Oligonucleotides and Immunoblotting. Methods Mol Biol 2016; 339 1421:35-44.

340 14. Zeng F, Peritz T, Kannanayakal TJ, Kilk K, Eiriksdottir E, Langel U, et al. A protocol for PAIR: 341 PNA-assisted identification of RNA binding proteins in living cells. Nat Protoc 2006; 1(2):920-927.

342 15. Leppek K, Stoecklin G. An optimized streptavidin-binding RNA aptamer for purification of 343 ribonucleoprotein complexes identifies novel ARE-binding proteins. Nucleic Acids Res 2014; 344 42(2):e13.

345 16. Simon MD, Wang CI, Kharchenko PV, West JA, Chapman BA, Alekseyenko AA, et al. The 346 genomic binding sites of a noncoding RNA. Proc Natl Acad Sci U S A 2011; 108(51):20497-20502.

347 17. Chu C, Qu K, Zhong FL, Artandi SE, Chang HY. Genomic maps of long noncoding RNA 348 occupancy reveal principles of RNA-chromatin interactions. Mol Cell 2011; 44(4):667-678.

349 18. Matia-Gonzalez AM, Iadevaia V, Gerber AP. A versatile tandem RNA isolation procedure to 350 capture in vivo formed mRNA-protein complexes. Methods 2017; 118-119:93-100.

351 19. McHugh CA, Guttman M. RAP-MS: A Method to Identify Proteins that Interact Directly with 352 a Specific RNA Molecule in Cells. Methods Mol Biol 2018; 1649:473-488. 
353

354

355

356

357

358

359

360

361

362

363

364

365

366

367

368

369

370

371

372

373

374

375

376

377

378

379

380

381

382

383

384

385

386

387

388

389

390

391

392

393

394

395

396

20. West JA, Davis CP, Sunwoo H, Simon MD, Sadreyev RI, Wang PI, et al. The long noncoding RNAs NEAT1 and MALAT1 bind active chromatin sites. Mol Cell 2014; 55(5):791-802.

21. Terns MP. CRISPR-Based Technologies: Impact of RNA-Targeting Systems. Mol Cell 2018; 72(3):404-412.

22. Kim DI, Roux KJ. Filling the Void: Proximity-Based Labeling of Proteins in Living Cells. Trends Cell Biol 2016; 26(11):804-817.

23. Trinkle-Mulcahy L. Recent advances in proximity-based labeling methods for interactome mapping. F1000Res 2019; 8.

24. Kim DI, Jensen SC, Noble KA, Kc B, Roux KH, Motamedchaboki K, et al. An improved smaller biotin ligase for BioID proximity labeling. Mol Biol Cell 2016; 27(8):1188-1196.

25. Branon TC, Bosch JA, Sanchez AD, Udeshi ND, Svinkina T, Carr SA, et al. Efficient proximity labeling in living cells and organisms with TurboID. Nat Biotechnol 2018; 36(9):880-887.

26. Lam SS, Martell JD, Kamer KJ, Deerinck TJ, Ellisman MH, Mootha VK, et al. Directed evolution of APEX2 for electron microscopy and proximity labeling. Nat Methods 2015; 12(1):51-54.

27. Ramanathan M, Majzoub K, Rao DS, Neela PH, Zarnegar BJ, Mondal S, et al. RNA-protein interaction detection in living cells. Nat Methods 2018; 15(3):207-212.

28. Mukherjee J, Hermesh O, Eliscovich C, Nalpas N, Franz-Wachtel M, Macek B, et al. beta-Actin mRNA interactome mapping by proximity biotinylation. Proc Natl Acad Sci U S A 2019; 116(26):12863-12872.

29. Abudayyeh OO, Gootenberg JS, Essletzbichler P, Han S, Joung J, Belanto JJ, et al. RNA targeting with CRISPR-Cas13. Nature 2017; 550(7675):280-284.

30. Cox DBT, Gootenberg JS, Abudayyeh OO, Franklin B, Kellner MJ, Joung J, et al. RNA editing with CRISPR-Cas13. Science 2017; 358(6366):1019-1027.

31. Konermann S, Lotfy P, Brideau NJ, Oki J, Shokhirev MN, Hsu PD. Transcriptome Engineering with RNA-Targeting Type VI-D CRISPR Effectors. Cell 2018; 173(3):665-676 e614.

32. Myers SA, Wright J, Peckner R, Kalish BT, Zhang F, Carr SA. Discovery of proteins associated with a predefined genomic locus via dCas9-APEX-mediated proximity labeling. Nat Methods 2018; 15(6):437-439.

33. Gao XD, Tu LC, Mir A, Rodriguez T, Ding Y, Leszyk J, et al. C-BERST: defining subnuclear proteomic landscapes at genomic elements with dCas9-APEX2. Nat Methods 2018; 15(6):433-436.

34. Yang LZ, Wang Y, Li SQ, Yao RW, Luan PF, Wu H, et al. Dynamic Imaging of RNA in Living Cells by CRISPR-Cas13 Systems. Mol Cell 2019; 76(6):981-997 e987.

35. Echols N, Harrison P, Balasubramanian S, Luscombe NM, Bertone P, Zhang Z, et al. Comprehensive analysis of amino acid and nucleotide composition in eukaryotic genomes, comparing genes and pseudogenes. Nucleic Acids Res 2002; 30(11):2515-2523.

36. Tourasse NJ, Li WH. Selective constraints, amino acid composition, and the rate of protein evolution. Mol Biol Evol 2000; 17(4):656-664.

37. Chao JA, Patskovsky Y, Patel V, Levy M, Almo SC, Singer RH. ZBP1 recognition of beta-actin zipcode induces RNA looping. Genes Dev 2010; 24(2):148-158.

38. Geuens T, Bouhy D, Timmerman V. The hnRNP family: insights into their role in health and disease. Hum Genet 2016; 135(8):851-867.

39. Pan F, Huttelmaier S, Singer RH, Gu W. ZBP2 facilitates binding of ZBP1 to beta-actin mRNA during transcription. Mol Cell Biol 2007; 27(23):8340-8351.

40. Chu C, Zhang QC, da Rocha ST, Flynn RA, Bharadwaj M, Calabrese JM, et al. Systematic 
bioRxiv preprint doi: https://doi.org/10.1101/2020.04.09.033290; this version posted April 9, 2020. The copyright holder for this preprint (which was not certified by peer review) is the author/funder. All rights reserved. No reuse allowed without permission.

discovery of Xist RNA binding proteins. Cell 2015; 161(2):404-416.

398

399 


\section{Figure legends}

401

402

403

404

405

406

407

408

409

410

411

412

413

414

415

416

417

418

419

420

421

422

423

424

425

426

427

428

429

430

\section{Figure 1. Design of CBRPP}

(A) Schematic representation of CBRPP approach. By fusing dCas13 and PBL enzyme together, dCas13, under the guidance of a specific crRNA, acts as an RNA tracker to bring PBL enzyme to the target RNA, then PBL enzyme biotinylates surrounding proteins of the target RNA, followed by streptavidin beads enrichment of biotinylated proteins and mass spectrometry.

\section{Figure 2. dRfxCas13d is not suitable for CBRPP to study RNA-protein} interactions

(A) Plasmids used in this figure. NLS: nuclear localization sequence; NES: nuclear export sequence; EGFP: enhanced green fluorescent protein; T2A: T2A self-cleaving peptide; HA: hemagglutinin tag.

(B) Upper: HEK293T cells were co-transfected with RfxCas13d/RfxCas13d-APEX2/APEX2-RfxCas13d and B4GALNT1 Rfx-crRNA to detect the mRNA level of B4GALNT1 by RT-qPCR after 48 hours. Rfx-NT1: non-targeting Rfx-crRNA 1; Rfx-NT2: non-targeting Rfx-crRNA 2. B4-Rfx-crRNA: B4GALNT1 Rfx-crRNA. Bottom: western blotting to measure the protein expression level of RfxCas13d, APEX2-RfxCas13d and RfxCas13d-APEX2.

(C) HEK293T cells were co-transfected with RfxCas13d and ACTB Rfx-crRNAs to detect the mRNA level of ACTB by RT-qPCR after 48 hours.

(D) Representative images for dRfxCas13d-APEX2 imaging with two crRNAs targeting ACTB mRNA in HEK293T. Mock: no treatment. Sodium arsenite: treating cells with $0.5 \mathrm{mM}$ sodium arsenite for 30 minutes. Stress granules are indicated by G3BP1 staining. Scale bars, $10 \mu \mathrm{m}$.

Figure 3. Transient transfection of dPspCas13b-APEX2 to identify RBPs of ACTB mRNA

(A) Plasmids used in this figure. P2A: T2A self-cleaving peptide; Linker: 3x(GGGGS), G: glycine, S: serine.

(B) HEK293T cells were co-transfected with PspCas13b/PspCas13b-APEX2 and ACTB Psp-crRNAs to detect the mRNA level of ACTB after 48 hours. Psp-NT1: non-targeting Psp-crRNA 1; Psp-NT2: non-targeting Psp-crRNA 2.

(C) Western blotting to measure the protein expression level of PspCas13b and PspCas13b-APEX2. 
431 (D) HEK293T cells were co-transfected with dPspCas13b-APEX2-NES and different ACTB 432 Psp-crRNAs. Upper: RT-qPCR analysis of ACTB mRNA level in cells. Bottom: western blotting 433 to measure the protein expression level of ACTB in cells.

434 (E) Workflow of transiently transfected dPspCas13b-APEX2 to capture the proteins that interact 435 with ACTB mRNA in HEK293T. Psp-NT1-2: non-targeting Psp-crRNA 1 and non-targeting 436 Psp-crRNA 2; ACTB-Psp-crRNA3-4: ACTB Psp-crRNA 3 and ACTB Psp-crRNA 4.

437 (F) Western blotting to detect the biotinylation activity of HEK293T cells co-transfected with 438 dPspCas13b-APEX2-NES and different Psp-crRNAs.

439 (G) Scatter plot showing the number of peptides per protein after $\log 2$ transformation in 440 non-targeting Psp-crRNA group (X-axis) and ACTB Psp-crRNA group (Y-axis) from mass 441 spectrometry proteomics data. The red dots in the scatter plot represent known RBPs of ACTB 442 mRNA in StarBase v2.0 database. The experiments were done in HEK293T transiently transfected 443 with dPspCas13b-APEX2 and Psp-crRNAs.

444 Figure 4. Using dPspCas13b-BioID2/TurboID/APEX2 inducibly expressing cell 445 lines to identify RBPs of ACTB mRNA

446 (A) Plasmids used in this figure.

447 (B) HEK293T cells were co-transfected with PspCas13b or 448 PspCas13b-APEX2/BASU/BioID2/TurboID and ACTB Psp-crRNAs to detect the mRNA level of 449 ACTB after 48 hours.

450 (C) Western blotting to test the inducible ability and the biotinylation activity of four stable 451 HEK293T cell lines for inducible expression of dPspCas13b-BioID2/TurboID/BASU/Apex2. Dox: 452 doxycycline.

453 (D) Timeline to capture the proteins that interact with ACTB mRNA using 454 dPspCas13b-BioID2/TurboID/Apex2 inducibly expressing cell lines.

455 (E) Western blotting to detect the expression level and biotinylation activity of cells collected from 456 (D).

457 (F) Scatter plot showing the number of peptides per protein after $\log 2$ transformation in 458 non-targeting Psp-crRNA group (X-axis) and ACTB Psp-crRNA group (Y-axis) from mass 459 spectrometry proteomics data. The red dots in the scatter plot represent known RBPs of ACTB 460 mRNA in StarBase v2.0 database. The experiments were done in dPspCas13b-BioID2 inducibly 461 expressing cell line. 


\section{Table legends}

464 Table 1. plasmids used in this paper

465 Table 2. crRNAs used in this paper

466 Table 3. qPCR primers used in this paper

467 


\section{Supplementary figure legends}

\section{Supplementary Figure 1}

470 (A) Work model of Tet-On 3G inducible expression system. Doxycycline binds the rtTA 471 transcription factor and allows it to bind DNA at the promoter. Gene expression is induced in the 472 presence of doxycycline. Reverse tetracycline-controlled transactivator (rtTA) is created by fusing 473 reverse Tet repressor (rTetR) with VP16. TRE: Tet response element. Dox: doxycycline, a analog 474 of tetracycline.

\section{Supplementary Figure 2}

476 Scatter plot showing the number of peptides per protein after $\log 2$ transformation in non-targeting 477 Psp-crRNA group (X-axis) and ACTB Psp-crRNA group (Y-axis) from mass spectrometry 478 proteomics data. The red dots in the scatter plot represent known RBPs of ACTB mRNA in 479 StarBase v2.0 database.

480 (A) The experiments were done in dPspCas13b-APEX2 inducibly expressing cell line.

481 (B) The experiments were done in dPspCas13b-TurboID inducibly expressing cell line. 
A
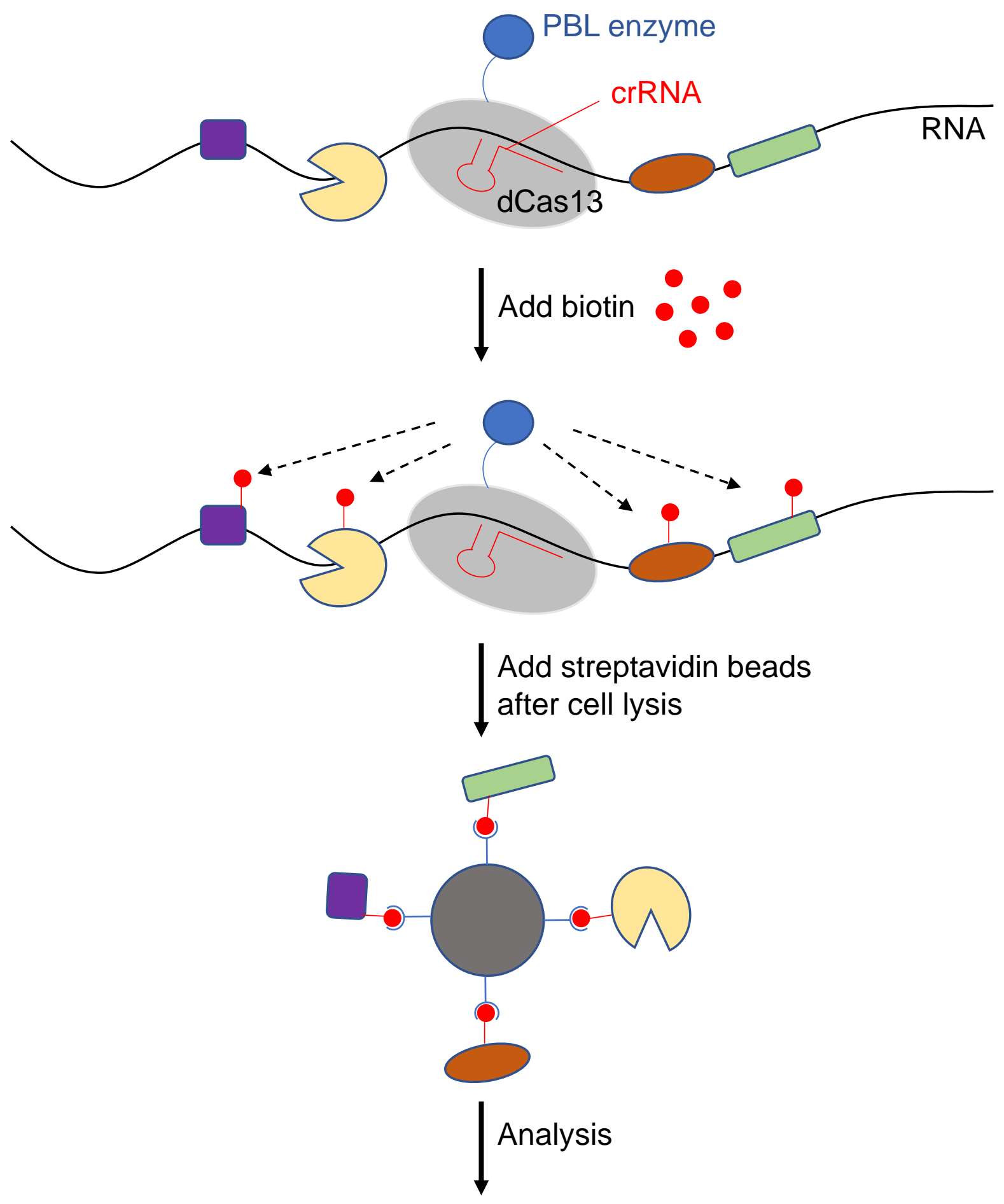

Mass spectrometry 
A

RfxCas13d

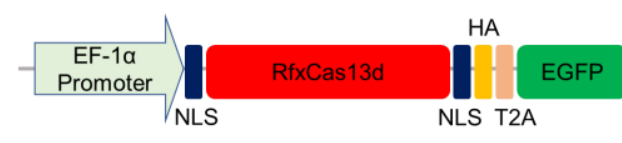

APEX2-RfxCas13d

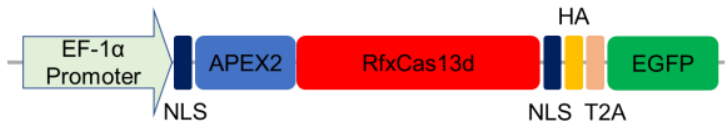

RfXCas13d-APEX2
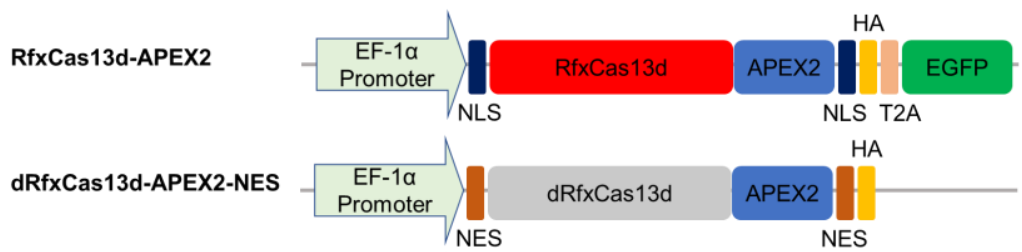

Rfx-crRNA

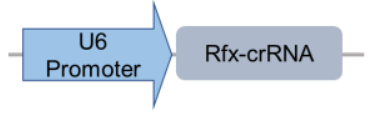

$\mathrm{B}$
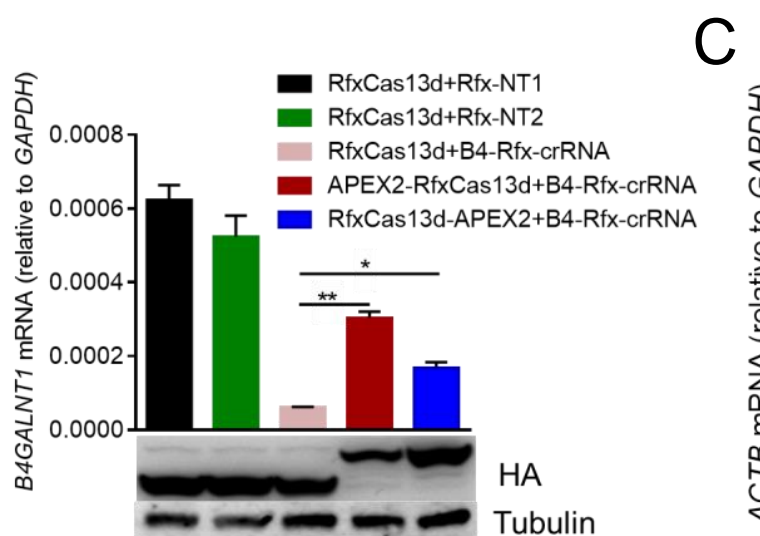

$\mathrm{D}$

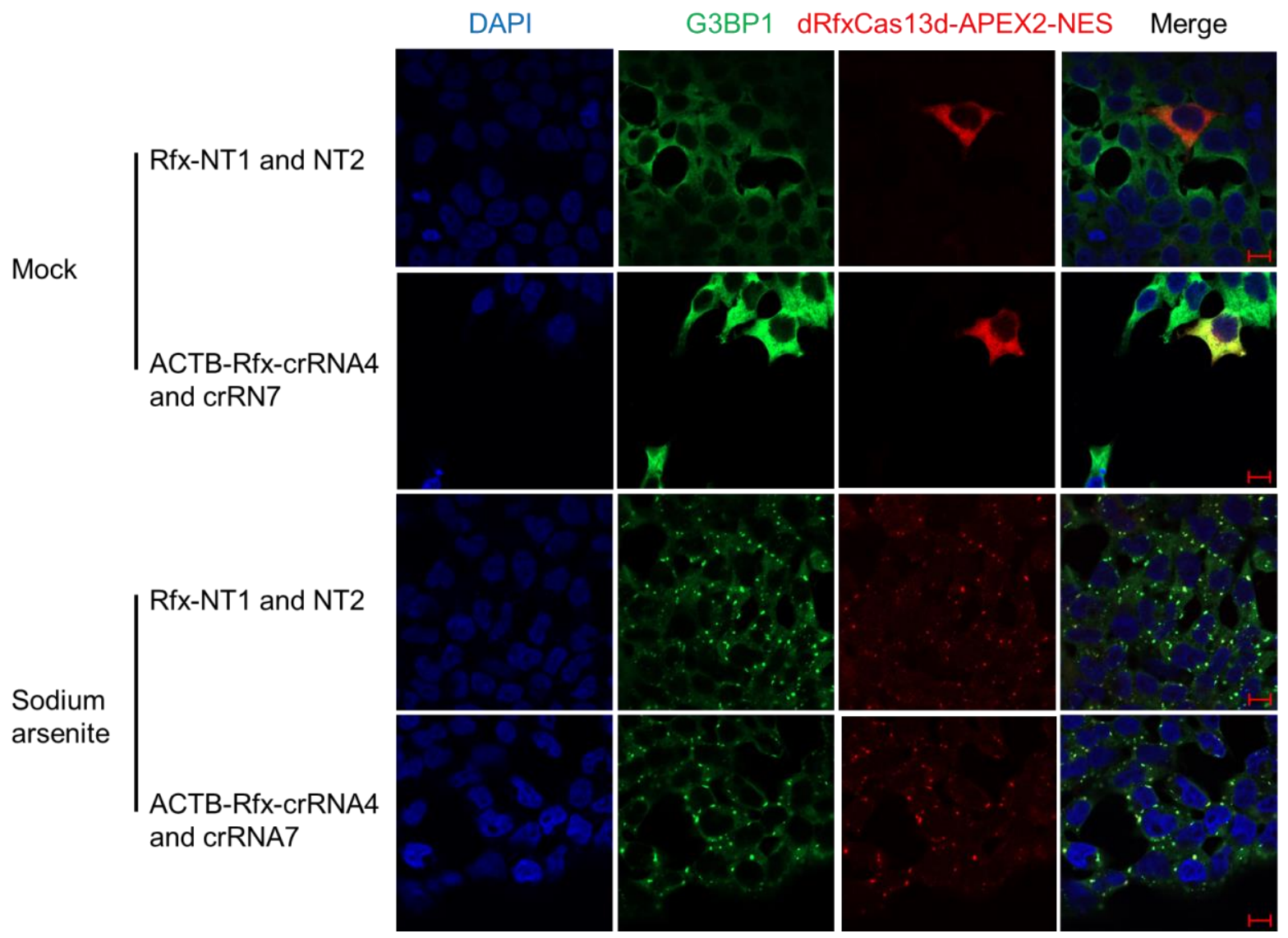

Figure 2. $d R f x C a s 13 d$ is not suitable for CBRPP to study RNA-protein interactions 
was not certified by peer review) is the author/funder. All rights reserved. No reuse allowed without permission.

A

PspCas13b

PspCas13b-APEX2

dPspCas13b-APEX2-NES

Psp-crRNA

C

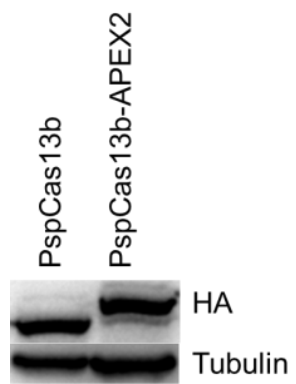

$\mathrm{F}$
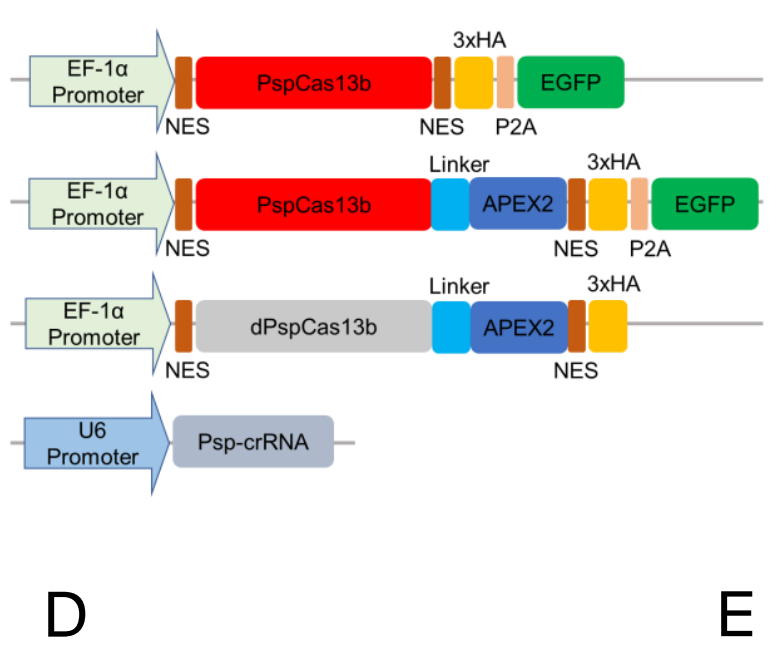

E
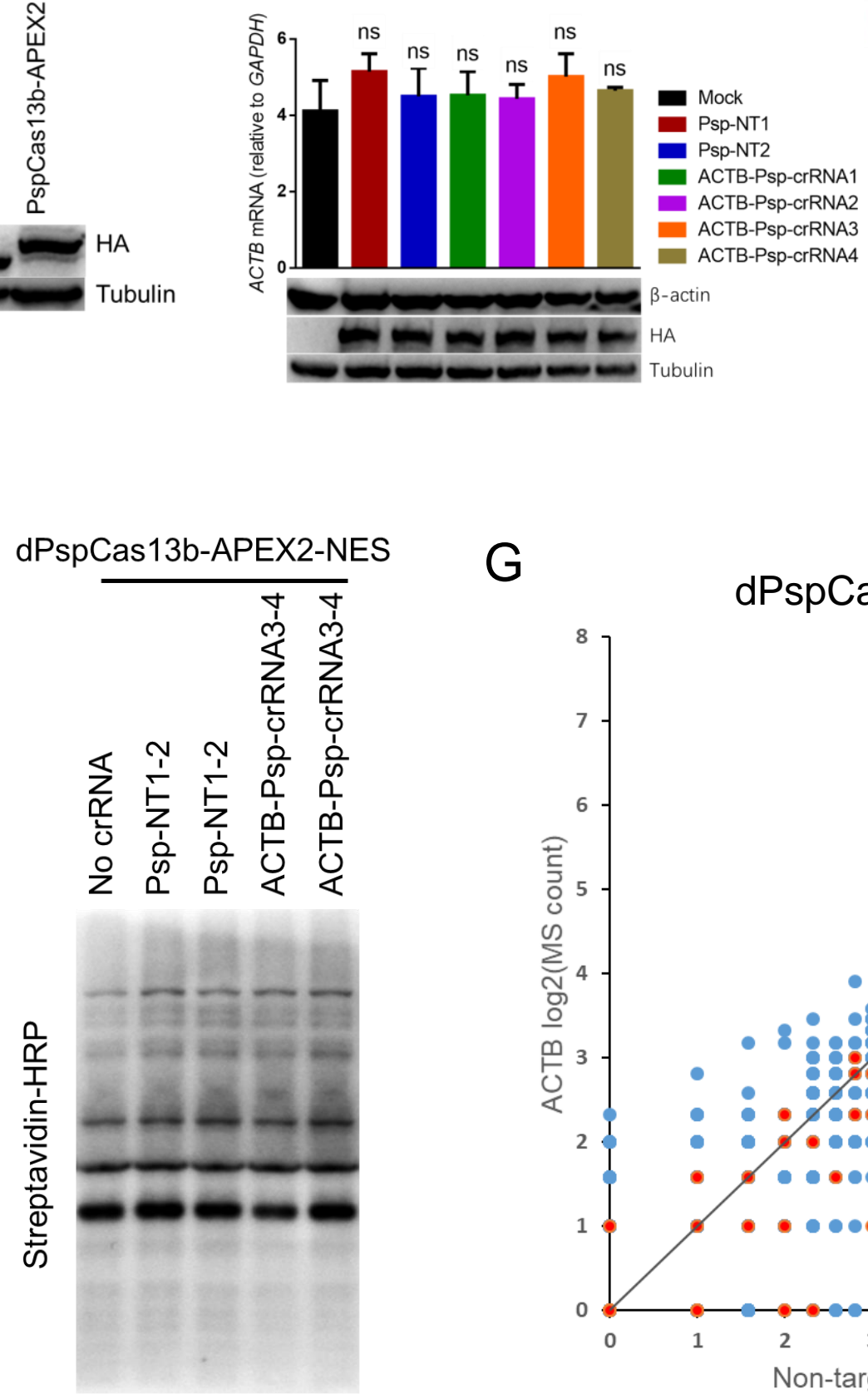

$G$
B

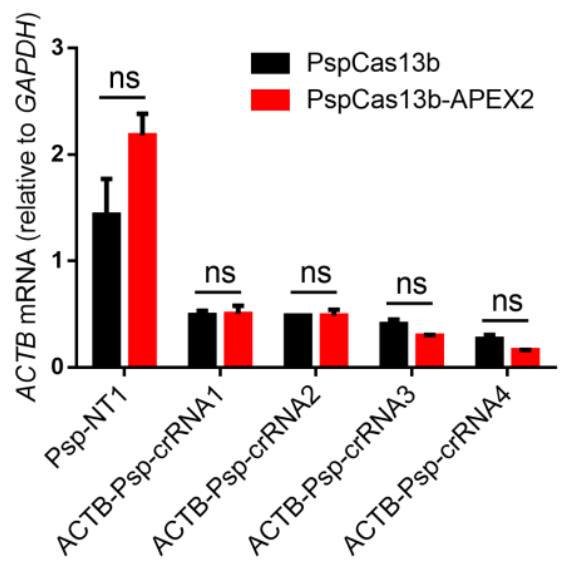

dPspCas13b-APEX2

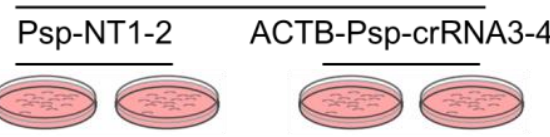

$\downarrow 24 \mathrm{~h}$

500uM Biotin-phenol 30min $1 \mathrm{mM} \mathrm{H}_{2} \mathrm{O}_{2} 1 \mathrm{~min}$

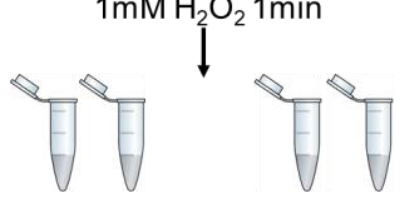

Cell lysis

Add streptavidin beads

Biotinylated protein enrichment

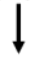

Mass spectrometry

dPspCas13b-APEX2

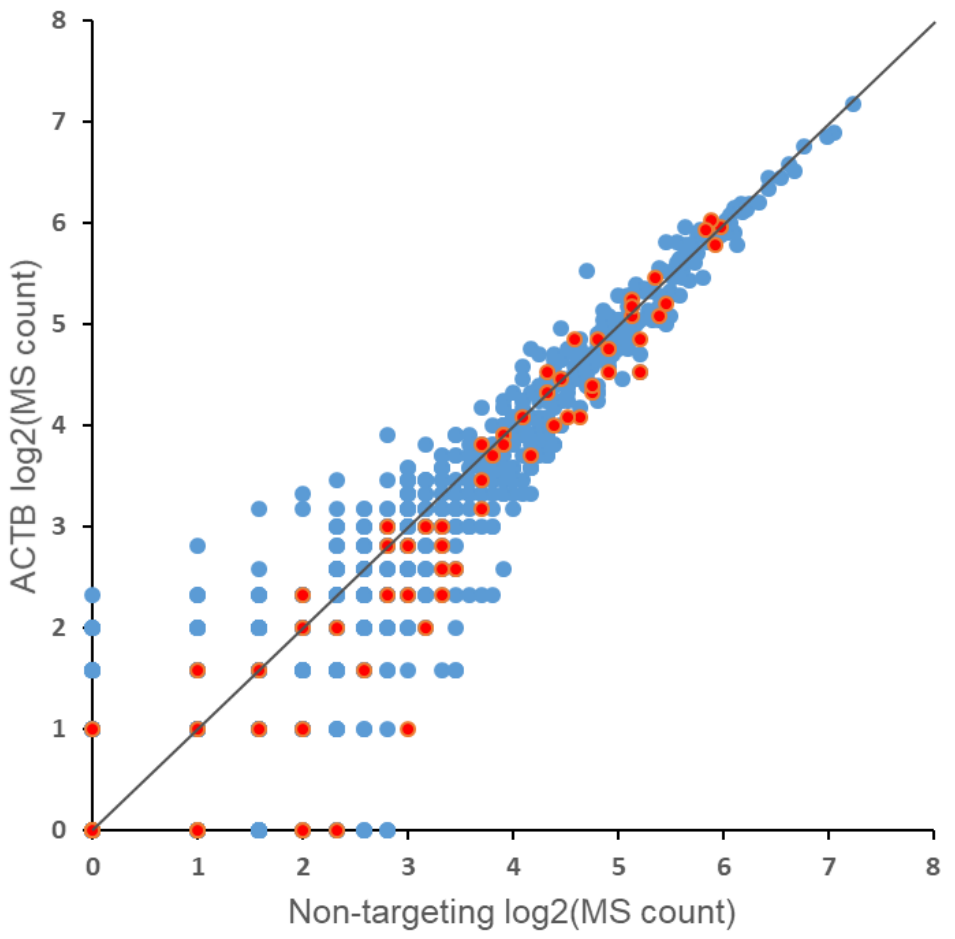

Figure 3. Transient transfection of dPspCas13b-APEX2 to identify RBPs of ACTB mRNA 
A $\quad$ B

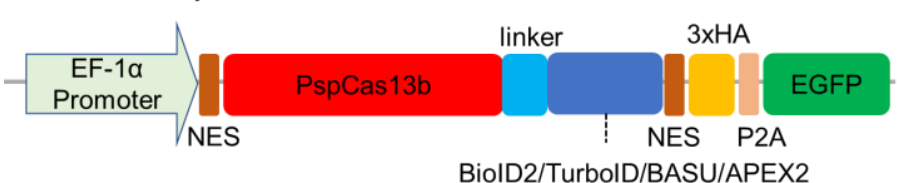

Inducible-dPspCas13b-BioID2/TurboID/BASU/APEX2-NES

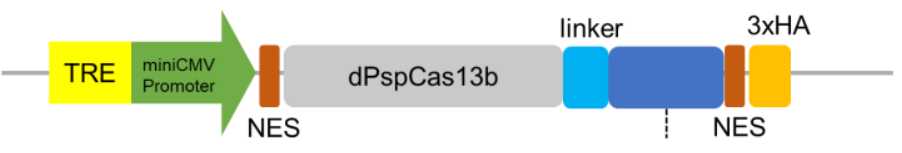

BiolD2/TurbolD/BASU/APEX2

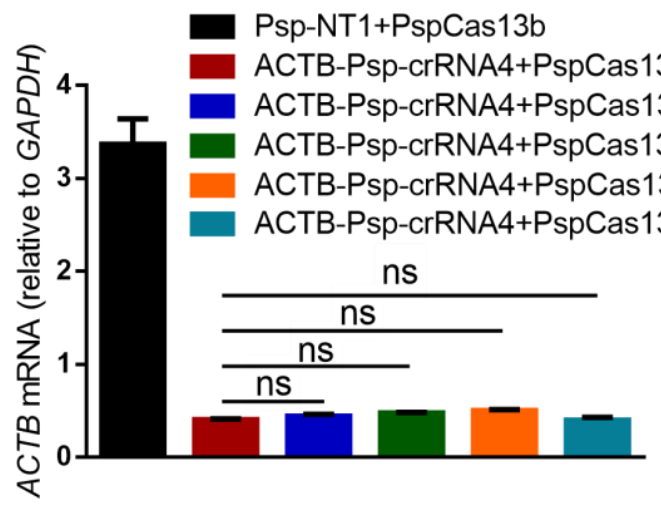

C

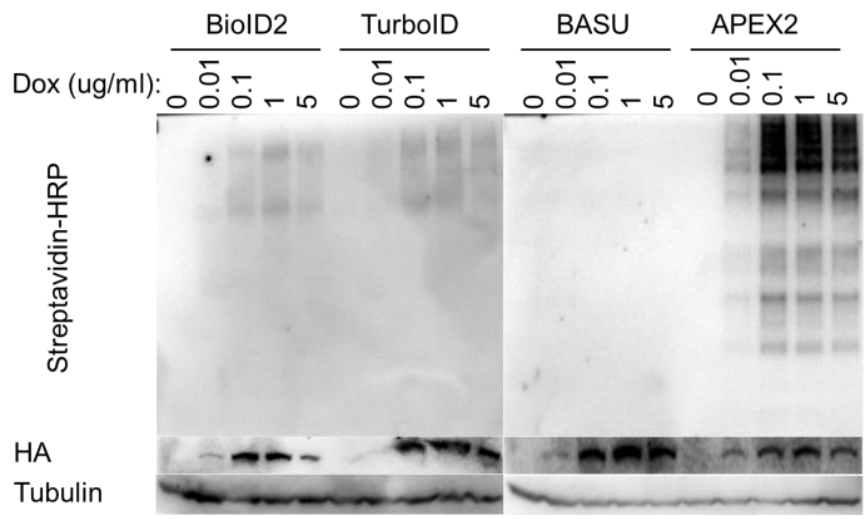

$\mathrm{D}$

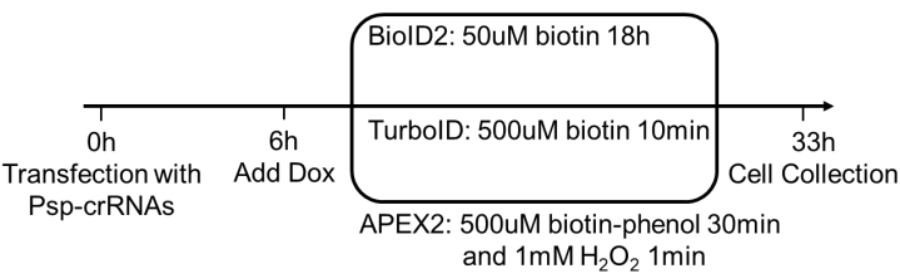

E

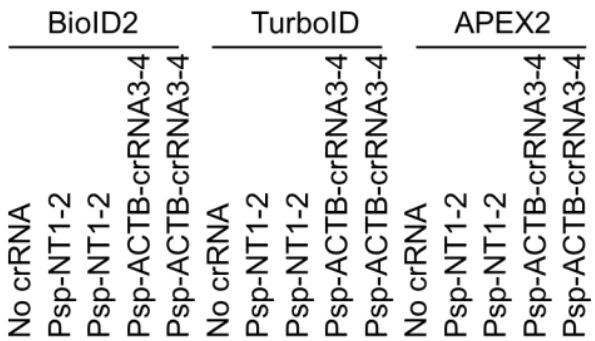

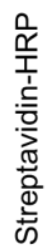

HA

Tubulin

\section{$\mathrm{F}$}

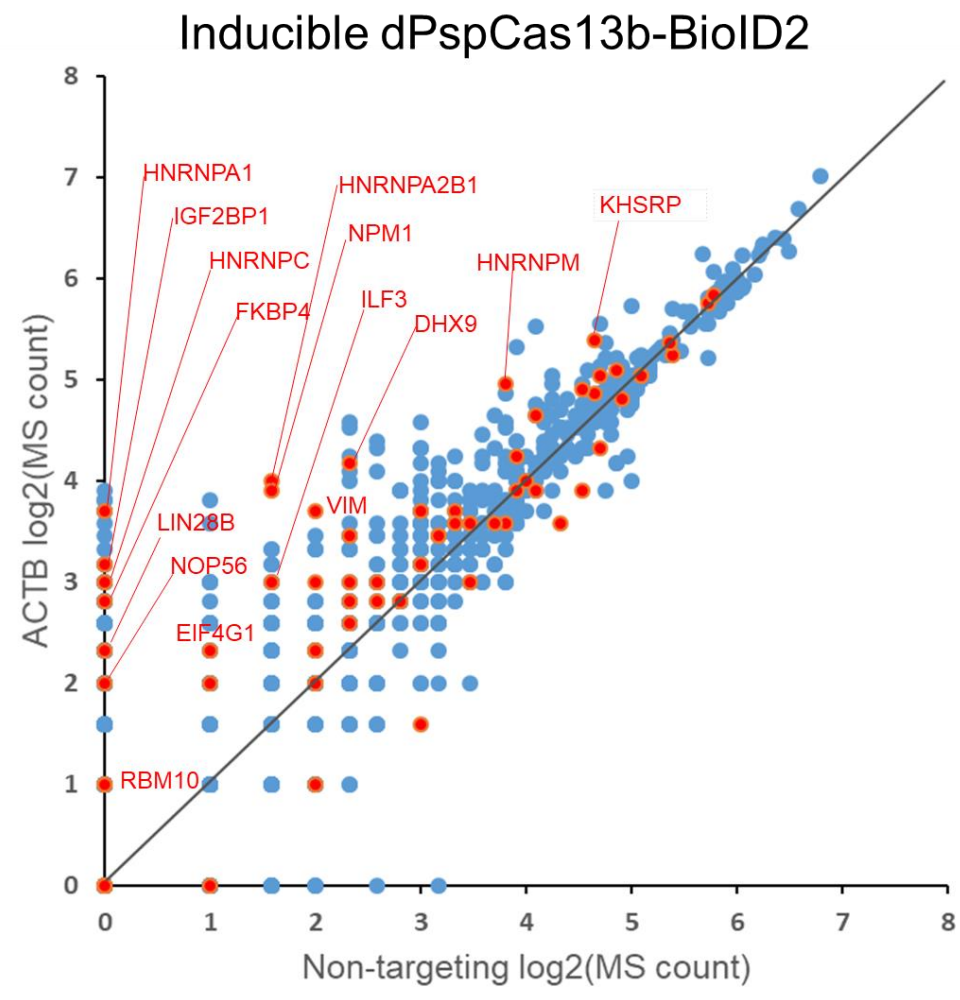

Figure 4. Using dPspCas13b-BiolD2/TurbolD/APEX2 inducibly expressing cell lines to identify RBPs of ACTB mRNA 